www.jmscr.igmpublication.org

Impact Factor (SJIF): 6.379

Index Copernicus Value: 71.58

ISSN (e)-2347-176x ISSN (p) 2455-0450

crossref DOI: https://dx.doi.org/10.18535/jmscr/v6i5.94

Journal Of Medical Science And Clinical Research

\title{
To find out the BMI Distribution among the Male Medical Students, to define-Obese and Non-obese
}

\author{
Authors \\ Dr M.P. Singh ${ }^{1}$, Dr Ashok Kumar ${ }^{2}$, Dr Manila Jain ${ }^{3}$ \\ ${ }^{1,2}$ Department of Physiology Amaltas Institute of Medical Sciences, Dewas, (M.P.), India \\ ${ }^{3}$ Index Medical College Hospital \& Research Centre, Indore, India
}

Corresponding Author

Dr Ashok Kumar

\begin{abstract}
Background: Obesity in young adults has risen to significant levels globally with serious public health consequences. In addition to cardiovascular, emotional and social issues, it poses a serious hazard to the basic health care delivery system. Unless this epidemic is contained at a war footing, the implications of this global phenomenon on future generations will be serious. The reversibility of this disease with suitable intervention strategies should be seen as an opportunity and efforts pursued with vigour. Also, the medical literature available in the Indian population is limited, only 3 similar studies and died are available in India and only 2 International studies available 1 of West Bank, Libiyal and another of Slovakia to hence the need for the present study arose.

Study Design: Cross-sectional and observational study.

Keywords: BMI, Male, Obese \& Non-Obese.
\end{abstract}

\section{Introduction}

Obesity is a medical condition in which excess body fat accumulates to the extent that it has an adverse effect on health, leading to reduced life expectancy and/or increased health problems (WHO 2000). It is defined by Body Mass Index (BMI) and further evaluated in terms of fat distribution via the waist-hip ratio and total cardiovascular risk factors (Sweeting, 2007). Body Mass Index (BMI) defines people as underweight when their BMI is $<18.5 \mathrm{~kg} / \mathrm{m}$, normal weight when BMI is between $18.5 \mathrm{~kg} / \mathrm{m}$ and $24.9 \mathrm{~kg} / \mathrm{m}$, overweight (pre-obese) when their BMI is between $25 \mathrm{~kg} / \mathrm{m}$ and $30 \mathrm{~kg} / \mathrm{m}$, and obese when it is greater than $30 \mathrm{~kg} / \mathrm{m}$ (WHO, 2000).
BMI provides a simple, convenient measurement of obesity ${ }^{1}$ The WHO Global Database on Body Mass Index (BMI) was developed as part of WHO's commitment to implementing the recommendations of the WHO Expert Consultation on Obesity: Preventing and Managing the Global Epidemic (Geneva, 3-5 June 1997)8", which identified the lack of nationally representative cross-sectional data as an obstacle for facilitating international comparisons of adulthood obesity rates, monitoring the magnitude of the current and future obesity problems, and evaluating the effectiveness of intervention strategies. $^{2}$ 


\section{Aims and Objectives}

The present study entitled "Comparative study of cardiovascular risk factors -Hypertension and lipid abnormalities, in Obese and Non-obese medical students of Index Medical College, Indore"., has been undertaken with the following aims and objectives:

1.To find out the BMI distribution among the male medical students, to define-Obese and Nonobese.

\section{Material and Methods}

The present study entitled "Comparative study of cardiovascular risk factors-Hypertension and lipid abnormalities, in Obese and Non-obese male medical students of Index Medical College, Indore will be conducted in the Department of Physiology, Index Medical College, Hospital and Research Centre, Indore for a duration of six months on young male medical students who are studying in Index Medical College.

Study Design: Cross-sectional and observational study.

Study Population: Male medical students of our institution.

Study Place: Department of Physiology, Index Medical College, Hospital \& Research Centre, Indore.

Size of the study population: Approx. 228 male medical students will be included after obtaining their written consent.

\section{Inclusion Criteria}

1. All male medical students of age group 18 and above.

2. Who willingly provide their voluntary written consent for participation in the study.

\section{Exclusion Criteria}

1.All female medical students. Students with known history of any cardiovascular condition.

Students not willing to provide their voluntary written consent.

\section{Methodology}

Male medical student filled up a questionnaire recording his age, diet and family history of hypertension. Body weight (to the nearest $0.5 \mathrm{~kg}$ ) standing motionless, feet $15 \mathrm{~cm}$ apart with minimum outwear (as culturally appropriate)and no footwear. Height (to the nearest $0.5 \mathrm{~cm}$ )with subject standing erect against a vertical surface with head in Frankfurt Plane. calculate by using the BMI 18.5), Weight and height was measured to calculate Body Mass Index (BMI) by FormulaWeight in $\mathrm{KG} /($ Height in Meter) - using the BMI criteria of $\mathrm{WHO} 2749$, the body types were categorized as underweight (BMI <18.5), normal (18.5-24.9), overweight (25.0-29.9) and obese (>30). Blood pressure was measured by Standard mercury Sphygmomanometer and classified as per the Seventh Report of the Joint National Committee where Pre-hypertension is systolic BP (SBP) of 120-139 mmHg or diastolic BP (DBP) of $80-89 \mathrm{mmHg}$ and Hypertension stage 1 is SBP of 140-159 or DBP of $90-99 \mathrm{mmHg}$. Subjects were informed about the study and their voluntary written consent was taken. Mean BP were computed for weight, height, BMI and blood pressure, data was analysed using chi-square test to find association between hypertension and variables (BMI of $<25 \&$ BMI $>25$, diet vegetarian and non-vegetarian and presence or absence of family history of hypertension). Those found to be significantly associated with hypertension $(\mathrm{P}<0.05)$ were then entered in Multiple logistic regression.

For serum lipid profile, Fasting venous samples were drawn from the antecubital space of the forearm between 9 am to 10 am with subjects being refrained from any consumption of food, alcohol or Caffeine containing beverages for $>=12$ hrs. Blood was collected in Patho. Laboratory of Index Hospital and Automated analysis was done for Triglyceride (TG),Cholesterol (C),Serum High Density Lipoprotein (S.HDL),Serum Low Density Lipoprotein(S.LDL), Serum Very Low Density Lipoprotein(S.VLDL. Normal values taken as follows TG- $<150 \mathrm{mg} / \mathrm{dl}$; C- 150-250 mg/dl ; 
S.HDL-35-55mg/dl; S.LDL- upto $120 \mathrm{mg} / \mathrm{dl}$; S.VLDL- more than 40

\section{Statistical Analysis}

For the present study, for comparing two variables, students ' $\mathrm{t}$ ' test - unpaired and paired will be used and for finding the association between the variables and hypertension, Pearson's chi-square test 21 and Multiple Logistic regression will be used. Any additional test as prescribed by the Statistician can be used on requirement or a test may also be changed as per the judgement of the statistician.

Table 01: The Relationship between Obesity with Serum Lipid Level and Blood Pressure

\begin{tabular}{|l|c|l|c|}
\hline I V & D V & $95 \%$ CI & P Value \\
\hline Obesity & Cholesterol & $(0.45-0.57)$ & 0.001 \\
\hline & Triglyceride & $(0.33-0.54)$ & 0.001 \\
\hline & $\begin{array}{c}\text { HDL } \\
\text { Cholesterol }\end{array}$ & $(0.38-0.18)$ & 0.001 \\
\hline & SBP & $(0.33-0.45)$ & 0.001 \\
\hline & DBP & $(0.18-0.36)$ & 0.001 \\
\hline
\end{tabular}

$\mathrm{IV}=$ Independent Variable, DV= Dependent Variable, 95\% $\mathrm{CI}=95 \%$ Confidence Interval., HDL-Cholesterol= High Density Lipoprotein Cholesterol.

Table shows positive and Significant $(\mathrm{p}=0.001)$ relationship of Obesity with Cholesterol, Triglyceride, SBP and DBP but Negative and significant relationship with HDL-cholesterol.

Table-02: The Relationship between Serum Lipid Level and Blood Pressure

\begin{tabular}{|l|c|c|c|}
\hline I V & D V & $95 \%$ CI & $\begin{array}{c}\text { P } \\
\text { Value }\end{array}$ \\
\hline Hypertension & Cholesterol & $(0.28-0.44)$ & 0 \\
\hline & Triglyceride & $(0.40-0.65)$ & 0.001 \\
\hline & $\begin{array}{c}\text { HDL } \\
\text { Cholesterol }\end{array}$ & $(0.66-0.29$ & 0.001 \\
\hline
\end{tabular}

IV- Independent Variable, DV= Dependent Variable, 95\% $\mathrm{CI}=95 \%$ Confidence Interval., HDL-Cholesterol= High Density Lipoprotein Cholesterol.

Table shows that Hypertension is Positively and Significantly $(\mathrm{p}=0.0001$ related to Triglyceride Weakly, Significant $(\mathrm{p}=\mathrm{o})$ relation with Cholesterol and Negatively and Significantly ( $p=0.0001$ related to HDL-Cholesterol

\section{Discussion}

In our study $18.42 \%$ students were obese while $\mathbf{6 9 . 7 3 \%}$ were Non-obese

Quazi Rais Ahmed et $\mathrm{al}^{3}$ in their study found out that $15 \%$ of male Medical students were obese Yasin I Tayem ${ }^{5}$ et al'2" in their study found out that $18.2 \%$ students were obese. These results are similar to our study RenuLohitashwa et al 28 in their study found out that $24.4 \%$ students were obese These results are higher than our study. LIFESTYLE In our study $58.77 \%$ students lead a Sedentary life while only $41.22 \%$ were Exercising regularly Yasin I Tayem ${ }^{5}$ et al127 in their study found out that $56.5 \%$ students were leading Sedentary life Quazi Rais ${ }^{3}$ Ahmed et al 129 in their study found out that $52.3 \%$ lead Sedentary life These results are similar to our study RenuLohitashwa ${ }^{4}$ et al128 in their study at Belgam found out that $70.03 \%$ students were leading sedentary life. These results are higher than our study. High prevalence of Hypertension and obesity in Belgaum study can be the fact that a much higher percentage $(70.03 \%)$ of their $\mathrm{e}$ leading a sedentary life as compared to our study group explained by the fact the students were leading a PERSONAL HABITS In our study $22.8 \%$ students were smokers, Quazi Rais Ahmed $^{3}$ et alin their study found out that $31.3 \%$ students were smokers Singh $\mathrm{SK}^{6}$ et al in their study "Smoking habits of the medical students found out that $30.7 \%$ were smokers. These results are similar to our study "Cardiovascular risk behaviour among students of a Medical College in Delhi found out that prevalence of smoking was $7 \%$ These results are less than our study Renu Lohitashwa $^{4}$ et al 128 in their study at Belgaum found out that $42 \%$ were smoking, Yasin $\mathrm{T}$ Tayem ${ }^{5}$ et al 12" in their study found out that 42.2\% were smokers Results are higher than our study and as smoking leads to deranged Profile, this can explain the high prevalence of hypertension in these Studies as compared to our study. In our study habit of Alcohol consumption was present in $14.03 \%$ Quazi Rais $\mathrm{Ahmed}^{3}$ et al. in their study found out that $16.33 \%$ consumed. 
These results are trends and acceptability Tayem et al in their study found out that $7.38 \%$ were consuming Alcohol This result is much result is much less than our study, which can be due to cultural prohibition to Alcohol consumption AMILY HISTORY (of Cardiovascular risk factors) In our study family history of Cardiovascular risk factors was present in $34.64 \%$ of students Which is much less than West Bank study of Yasin I Tayem ${ }^{5}$ et all21 (68.33\%) and Belgaum study of Renu Lohitashwa ${ }^{4}$ et al (72.03\%) while similar to Bareilly study of Quazi Rais Ahmed $^{3}$ et all29 (28\%), which can be due to regional eating habits, lifestyle and genetic factors.

\section{Diet}

In our study $14.49 \%$ vegetarian students were hypertensive while $14.5 \%$ Non-vegetarian were hypertensive In Delhi study by $120 \quad 15.2 \%$ vegetarian and $33.5 \%$ Non vegetarian were hypertensive Christopher $\mathrm{L} \mathrm{Melby}^{7}$ et al in their study "Blood pressure and blood lipids long vegetarian, semi vegetarian and non vegetarian African Americans" und out that $16 \%$ vegetarian and $31.1 \%$ Non-vegetarian were hypertensive Appleby $\mathrm{PN}^{8}$ et al in their study "Hypertension and blood pressure among meat eaters, vegetarian eaters, vegetarians and vegans in EPIC-Oxford" found out that $41.3 \%$ Non-vegetarian were hyperter Christopher $\mathrm{L}^{7} \mathrm{Me}$ population and $31.1 \%$ Christopher L Melby ${ }^{7}$ et al in their study found out that $16 \%$ vegetarian ton and $31.1 \%$ Nonvegetarian population were Hypertensive. These results are study for non-vegetarian and frequency of Its are similar to our study for vegetarians but much more than our on-vegetarians which can be due to Type of meat, portion of meat frequency of having Non-veg diet in Western population

\section{Socioeconomical Status (Ses)}

In our study $61.4 \%$ students belongs to High SES while $18.4 \%$ belonged to Middle SES; these findings are similar to study by Renu Lohitashwa ${ }^{4}$ et al $28(70.2 \%)$ and Tenzing Kyizom 123
(59.8\%) These findings associate the eating habits and sedentary lifestyle of affluent class with increasing prevalence of cardiovascular risk factors.

The respective P-values and Odds Ratio for BMI, diet and family history. All three variables were found to be significant after the application of Chi-square test. After applying multiple logistic regression, only BMI \& diet was found to be significantly associated with hypertension.

\section{Conclusion}

Obesity is a major heal major health hazard all over the world and becoming a major Health threat among both the sexes and all age groups. Substantial proportions of young of adult medical students medical students are pre hypertensive and obese. Our results highlight the necessity to institute effective prevention and the promotion programs targeting younger age groups. In view of the fact at medical students are not representative of the general population, studies should be extended to the young adult population and investigate the presence of obesity and major CVD risk factors and their trends over time. Unhealthy behavioural practices are present and may progress as student advance through medical college. Developing strategies targeting at these risk behaviours and determining factors is necessary to promote healthy life style among medical students. Modifiable cardiovascular risk behaviours are widely prevalent among medical students and increase with years spent in the medical college. Promotion of supportive environment for strengthening student-based approaches and strategic delivery of health education is essential to target these risk behaviours among our future doctors.

\section{References}

1. Zinner SH, Levy PS, Kass EH. Familial Aggregation of BloodPressure in Childhood. N Engl J Med 1971; 284:401404. 
2. Sacks FM, B Rosrier, EH Kass Blood pressure in vegetarians. Am J Epidemiol 1974; 100: 390-398.

3. QuaziRais Ahmed, Rajesh Kumar Seth Prevalence of hypertension, obesity, anaemia among first yr Dental students in Barelliey. Indian J Physiol Pharmacol 2013;5(15):2241-2243.

4. Renu Lohitashwa, Parwati Patil Prevalence and trends of obesity and hypertension among young Medical students- a crosssectional study. Int J Biol Res 2013;4(4):3540-3543

5. Yasin I. Tayem, Nagham A. Yaseen, Wiam T. Khader, Prevalence and risk factors of obesity and hypertension among students at a central university in the West Bank. Libyan J Med. 2012; 7: 10.3402/ljimv7io. 19222.

6. Singh SK, Narang RK, Chandra S, Chaturvedi PK, Dubey AL.Smoking habits of the medical students. Indian J Chest Dis Allied Sci. 1989;31:99-103. [PubMed].

7. Melby CL, Toohey ML, Cebrick J. Blood pressure and blood lipids among vegetarian, semi vegetarian and nonvegetarian African Americans. Am J Clin Nutr1994; 59(1): 103-109.

8. Apple by PN, Davey GK, Key TJ. Hypertension and blood pressure among meat eaters, fish eaters, vegetarians and vegans in EPIC-Oxford. Public Health Nutr2002; (5): 645-654. 